\title{
Comparing two methods of visual abstraction to human data
}

\author{
FREDERICK J. BREMNER and STEVE GOTTS \\ Trinity University, San Antonio, Texas
}

\begin{abstract}
The ability to "visually abstract" a given pattern with a neural network and abstract the same pattern by using a regression/correlation analysis was investigated. Both methods were compared with human subjects performing the same task. To visually abstract a particular shape, both quantitative methods broke the shape down into its linear, quadratic, and cubic components. Using an IBM-compatible personal computer, 10 test patterns were analyzed with a neural network (designed using Brainmaker Professional and trained with known linear, quadratic, and cubic shapes) and a regression/correlation model (designed using Lotus 1-2-3). The 10 test patterns were also analyzed by 22 human subjects. The neural network data were found to be highly correlated with the human data $[r(8)=.90, p<.01]$. The regression/correlation model's data were also found to be significantly correlated with the human data $[r(8)=.77, p<.01]$. These findings demonstrate the successful modeling of Rumelhart's (1991) regression/correlation approach to visual abstraction.
\end{abstract}

The visual system is one of the most complicated and intriguing physiological systems in the human body. Some researchers have studied how vision actually occurs and have developed interesting theories from their findings. One such theory, presented by Marr (1982), claims that the basic task of vision is one of two- to three-dimensional mapping. He argued that as visual stimuli are processed, the brain abstracts the perceived scene, degree by degree (in three stages), until the image is broken down into its most essential and necessary components. Other investigators (Yonas, Craton, \& Thompson, 1987) have studied the factors of object boundaries, overlapping images, and depth in the abstraction of the visual field. Another theorist, Rumelhart (1991), suggests a multilayered neural network as a model for visual abstraction. In his model, there are three backpropagation networks: one for linear components, one for quadratic components, and one for cubic components.

In the present study, we built on Rumelhart's strategy by abstracting a given shape into its linear, quadratic, and cubic components and empirically determining values for each component. Once each of these values was computed, the shape was classified as "more linear," "more quadratic," or "more cubic," depending on which value had the greatest magnitude.

Two distinctly separate methods were employed to perform this abstraction: (1) a connectionist, backpropagation, neural network, and (2) a multiple regression/correlation statistical program written in the context of a spreadsheet. Both of the models were compared with human subjects performing the same task (they were given definitions of

Address correspondence to F. J. Bremner, Department of Psychology, Trinity University, 715 Stadium Drive, San Antonio, TX 78212 (phone: 210-736-8383). linear, quadratic, and cubic shapes with hallmark examples of each). The research task was to determine if the two computer methods could successfully model the functioning of humans performing the same kind of "visual abstraction," given the same 10 test patterns. We believed that both methods would successfully model the human subjects on the task. We measured our dependent variable "degree"' ( 1 for linear, 2 for quadratic, and 3 for cubic) at the three levels of the independent variable "method" (the first level being the neural network, the second being the statistical program, and the third being the human subjects) for each of the 10 unique test patterns.

\section{METHOD}

\section{Subjects}

Twenty-two students from the Trinity University Human Subjects Pool served as voluntary participants. There were no subject restrictions. Volunteers (students enrolled in lower division psychology classes) received extra credit toward their grades for their participation in the experiment.

\section{Patterns}

The stimuli used in the experiment were 10 unique test patterns that we designed. The patterns were constructed in a $20 \times 20$, twodimensional, Cartesian $(x, y)$ coordinate system and were designed to span all four quadrants of the Cartesian plane. The desire for uniqueness played a very important part in the designing of the set of 10 test shapes. However, we also wanted to make sure that each pattern in the test set was modeled after one of the three polynomial functions. Three of the 10 patterns were designed to be essentially linear, 4 were designed to be essentially quadratic, and 3 others were designed to be essentially cubic.

\section{Apparatus}

An IBM-compatible personal computer was used to implement both simulations of the human visual system and to present the 10 stimuli to the human subjects. We used Brainmaker Professional 
1

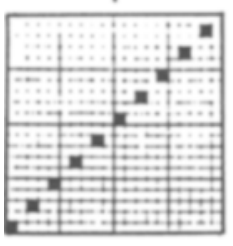

4

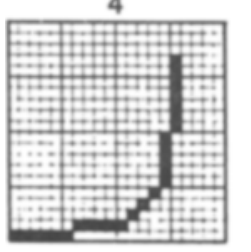

7

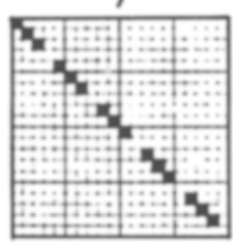

2

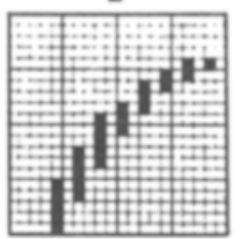

5

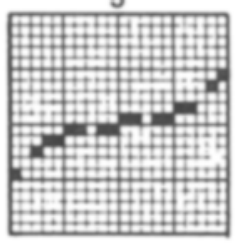

8

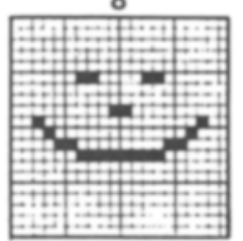

10

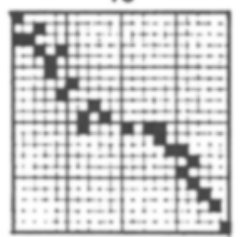

3

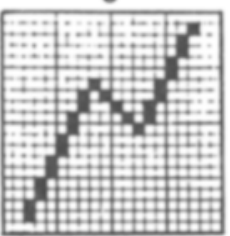

6

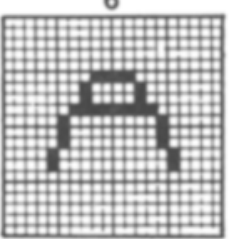

9

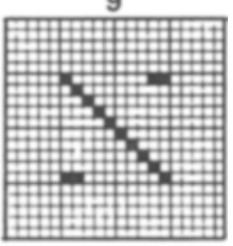

using Pearson product-moment correlations, then with the $x^{2}$ scores and $x^{3}$ scores (Cohen \& Cohen, 1983).

Human subjects. Two groups of subjects ( 11 in each group) participated in the experiment. One group performed the task in a room designated for the experiment. Each subject in this group performed the experiment in an isolated environment, and only 1 subject was in the room at any one time. The second group of subjects performed the task in a classroom (these subjects were actually members of a lower division psychology class and participated in the experiment during their regular class time). We organized these two groups of subjects and their "degree" measurements into a between-within subjects design. Using SPSS ${ }^{\star}$ and the MANOVA command, we established that there were no significant main effects for the between-subject variables of group and gender. This allowed us to pool the subjects into one group.

\section{Procedure}

Brainmaker. To evaluate each of the 10 test patterns using Brainmaker, we designed a run file (a file in which the input shapes have no "teacher" value) consisting of the 10 shapes. We then ran Brainmaker, training the network with the definition file. Using the singlestep flag in the "options" menu, we executed the run file containing our test data and recorded the output values $\left(x, x^{2}\right.$, and $\left.x^{3}\right)$ for each shape. A degree (of match) value of 1,2 , or 3 was assigned to each shape, depending on which output value was the largest (1 corresponds to $x, 2$ to $x^{2}$, and 3 to $x^{3}$ ). A score of 1 signifies that the shape is basically linear, a 2 signifies that it is mostly quadratic, and $a$ indicates an overall cubic nature. These degree values were also recorded for all 10 shapes.

Lotus 1-2-3. Each of the 10 test patterns was converted into sets of $(x, y)$ pairs (one pair corresponds to a cell in the $20 \times 20$ matrix representation). The program returned absolute values for $r(y x)$, $r\left(y x^{2}\right)$, and $r\left(y x^{3}\right)$. These correlation coefficients were then recorded for each of the 10 shapes. A degree value of either 1, 2, or 3 was assigned to each shape, depending on which absolute $r$ value was the greatest [1 corresponds to $r(y x), 2$ to $r\left(y x^{2}\right), 3$ to $\left.r\left(y x^{3}\right)\right]$. These degree values were also recorded for all of the test patterns.

Human subjects. The 10 test shapes were presented to all 22 subjects. The subjects were asked to classify each test shape as "more linear," "more quadratic," or "more cubic" on the basis of their perception of the given shapes. As a result, each of the 10 test shapes had 22 scores associated with it. To generalize how the subjects perceived each shape, we took the mean of the 22 degree measures for each shape. These means were recorded for all of the 10 test patterns.

The degree values generated by Brainmaker were then compared with the human subject mean degree values by correlating the two sets of data with each other, using Pearson product-moment correlations. The degree values for the spreadsheet model were compared with the human data in the same way.

\section{RESULTS}

Table 1 shows the empirical evaluation of the 10 test shapes by the Brainmaker neural network. Note that the reported values for $x, x^{2}$, and $x^{3}$ were "strength" values for the 10 shapes. Also note that the strength values are between 0 and 1 and that they determine the degree values on the right.

Table 2 shows the information supplied by the Lotus 1-2-3 program upon analyzing the 10 test shapes. The $r$ values in Table 2 indicate the strength of the linear, quadratic, and cubic components for each of the 10 test shapes. The degree values, as in Table 1, are located at the far right 
Table 1

Brainmaker Strength and Degree Values

\begin{tabular}{ccccc}
\hline Shape & $x$ & $x^{2}$ & $x^{3}$ & Degree Value \\
\hline 1 & .995 & .015 & .032 & 1 \\
2 & .977 & .002 & .196 & 1 \\
3 & .006 & .000 & .996 & 3 \\
4 & .235 & .348 & .100 & 2 \\
5 & .055 & .000 & .986 & 3 \\
6 & .033 & .999 & .005 & 2 \\
7 & .999 & .000 & .037 & 1 \\
8 & .063 & .999 & .000 & 2 \\
9 & .999 & .001 & .005 & 1 \\
10 & .000 & .000 & .998 & 3 \\
\hline
\end{tabular}

Table 2

Lotus 1-2-3 $r$ and Degree Values

\begin{tabular}{ccccc}
\hline Shape & $r(y x)$ & $r\left(y x^{2}\right)$ & $r\left(y x^{3}\right)$ & Degree Value \\
\hline 1 & 1.000 & .368 & .906 & 1 \\
2 & .945 & .133 & .828 & 1 \\
3 & .884 & .440 & .969 & 3 \\
4 & .821 & .184 & .606 & 1 \\
5 & .940 & .254 & .980 & 3 \\
6 & .204 & .727 & .685 & 2 \\
7 & 1.000 & .374 & .910 & 1 \\
8 & .000 & .010 & .002 & 2 \\
9 & .068 & .027 & .004 & 1 \\
10 & .962 & .340 & .937 & 1 \\
\hline
\end{tabular}

Table 3

Degree Values of Both Computer Models With Human Means

\begin{tabular}{cccc}
\hline Shape & Brainmaker & Lotus 1-2-3 & Humans \\
\hline 1 & 1 & 1 & 1.000 \\
2 & 1 & 1 & 1.864 \\
3 & 3 & 3 & 2.909 \\
4 & 2 & 1 & 2.182 \\
5 & 3 & 3 & 3.000 \\
6 & 2 & 2 & 2.046 \\
7 & 1 & 1 & 1.000 \\
8 & 2 & 2 & 2.000 \\
9 & 1 & 1 & 1.091 \\
10 & 3 & 1 & 2.273 \\
\hline
\end{tabular}

and are determined by comparing the three $r$ values for each shape.

The Pearson product-moment correlation of the human data with the Brainmaker data revealed a substantial relationship $[r(8)=.90, p<.01]$. Similarly, the correlation of the human data with the Lotus 1-2-3 data also revealed a substantial relationship $[r(8)=.77, p<.01]$.

\section{DISCUSSION}

The current data establish a successful modeling of Rumelhart's (1991) regression/correlation approach to visual abstraction with both neural network and spreadsheet models. The high correlations between the human data and the models support our premise that human vision can be simulated with this three-component approach. It is probably not significant that the two correlations are more than .10 apart. In order to test that there is a nontrivial difference between our two significant $r$ values, we need to increase the degrees of freedom. While this can easily be achieved by adding more test shapes to our existing set, it was never the purpose of this project to attempt to discriminate between the spreadsheet and the neural network model.

One topic that should be addressed is the simplistic nature of the 10 test shapes. In designing the test shapes, we focused on equal representation of each of the three types of shape. To ensure that each classification was equally represented in the test set, we had to stay reasonably close to a linear, quadratic, or cubic shape. With this approach, we were able to see that the models had no deficiencies in discriminating any one type of classification (such as an inability to see shapes as cubic). If the models could only recognize linear shapes, they would be less valid. To guard against that, we designed each shape to closely resemble one of the three basic classifications of the shapes. The criticism of this approach is that the test shapes do not possess the same level of complexity of objects that we actually observe in the real world, although Test Shapes 6, 8, and 9 were more complex. In future studies, more shapes will be used to correspond closely with objects actually present in the real world. The next step in the research is to test a neural network composed of three subnetworks: one for linear, one for quadratic, and one for cubic abstraction of complex shapes.

\section{REFERENCES}

California Scientific Software (1990). Brainmaker professional [Computer program]. Grass Valley, CA: Author.

COHEN, J., \& COHEN, P. (1983). Applied multiple regression/correlation analysis for the behavioral sciences. Hillsdale, $\mathrm{NJ}$ : Erlbaum.

Lotus DeVElopMent CoRPoRATION (1985). Lotus 1-2-3 [Computer program]. Cambridge, MA: Author.

MARR, D. A. (1982). Vision. San Francisco: W. H. Freeman

RUMELhART, D. (1991, November). A statistical account of learning. Keynote address, Conference of the Society for Computers in Psychology, San Francisco.

Yonas, A., Craton, L. G., \& Thompson, W. B. (1987). Relative motion: Kinetic information for the order of depth at an edge. Perception \& Psychophysics, 41, 53-59. 